\title{
Correction to: Learning curves of two different techniques for the intra-articular injection of the knee joint under fluoroscopic guidance
}

\author{
Paolo Simoni $^{1,3} \cdot$ Olivier Malaise $^{3} \cdot$ Mounia El Hachemi $^{2} \cdot$ Angelo Tromba $^{2} \cdot$ Grammatina Boitsios $^{1}$
}

Published online: 17 February 2018

(c) Italian Society of Medical Radiology 2018

\section{Correction to: La radiologia medica \\ https://doi.org/10.1007/s11547-017-0847-2}

The published version of this article contained a mistake. In Table 1 of the original article, the numbers of successful injections and failed injections were not correct. The correct Table 1 should read as given below:

The original article can be found online at https://doi.org/10.1007/ s11547-017-0847-2.

Paolo Simoni

paoloemiliosimoni@gmail.com

1 "Reine Fabiola" Children's University Hospital, Université Libre de Bruxelles (ULB), 15, Avenue Jean Joseph Crocq, 1020 Brussels, Belgium

2 Radiology Department, CHU de Liège, CHU du Sart Tilman, Bât. 35, 4000 Liège, Belgium

3 Rheumatology Department, CHU de Liège, CHU du Sart Tilman, Bât. 35, 4000 Liège, Belgium 
Table 1 Comparison between supine and lateral techniques

\begin{tabular}{|c|c|c|c|c|}
\hline Variable & Overall $(n=177)$ & $\begin{array}{l}\text { Supine technique } \\
(n=101)\end{array}$ & $\begin{array}{l}\text { Lateral technique } \\
(n=76)\end{array}$ & $p$ value \\
\hline Operator & & & & 0.92 \\
\hline Operator 1 & $56(31.6)$ & $33(32.7)$ & $23(30.3)$ & \\
\hline Operator 2 & $63(35.6)$ & $36(35.6)$ & $27(35.5)$ & \\
\hline Operator 3 & $58(32.8)$ & $32(31.7)$ & $26(34.2)$ & \\
\hline \multicolumn{5}{|l|}{ Sex } \\
\hline Female & $72(40.7)$ & $40(39.6)$ & $32(42.1)$ & 0.74 \\
\hline Male & $105(59.3)$ & $61(60.4)$ & $44(57.9)$ & \\
\hline Age (years) & $45.2 \pm 15.0$ & $45.6 \pm 14.7$ & $44.6 \pm 15.5$ & 0.65 \\
\hline Weight (kg) & $77.9 \pm 14.8$ & $78.5 \pm 13.6$ & $76.9 \pm 16.4$ & 0.48 \\
\hline Height (cm) & $172.1 \pm 9.76$ & $172.5 \pm 9.06$ & $171.5 \pm 10.7$ & 0.50 \\
\hline BMI $\left(\mathrm{kg} / \mathrm{m}^{2}\right)$ & $26.3 \pm 4.48$ & $26.4 \pm 4.10$ & $26.1 \pm 4.96$ & 0.69 \\
\hline Injection & & & & 0.027 \\
\hline Successful & $151(85.3)$ & $81(80.2)$ & $70(92.1)$ & \\
\hline Failed & $26(14.7)$ & $20(19.8)$ & $6(7.9)$ & \\
\hline NSAID & & & & 0.50 \\
\hline Yes & $150(84.8)$ & $84(83.2)$ & $66(86.8)$ & \\
\hline No & $27(15.3)$ & $17(16.8)$ & $10(13.2)$ & \\
\hline Previous trauma or surgery & & & & 0.081 \\
\hline No & 109 (61.9) & $57(56.4)$ & $52(69.3)$ & \\
\hline Yes & $67(38.1)$ & $44(43.6)$ & $23(30.7)$ & \\
\hline VAS before injection & $2.01 \pm 2.55$ & $2.23 \pm 2.55$ & $1.71 \pm 2.54$ & 0.18 \\
\hline VAS injection & $4.10 \pm 2.83$ & $4.36 \pm 2.68$ & $3.76 \pm 3.00$ & 0.16 \\
\hline VAS difference & $2.10 \pm 2.63$ & $2.13 \pm 2.31$ & $2.05 \pm 3.03$ & 0.85 \\
\hline$(p \text { value })^{*}$ & $(<0.0001)$ & $(<0.0001)$ & $(<0.0001)$ & \\
\hline Fluoroscopy (s) & $7.06 \pm 4.88$ & $7.58 \pm 5.26$ & $6.37 \pm 4.25$ & 0.10 \\
\hline Extravasation & & & & $<0.0001$ \\
\hline No & $95(53.7)$ & $34(33.7)$ & $61(80.3)$ & \\
\hline Yes & $82(46.3)$ & $67(66.3)$ & $15(19.7)$ & \\
\hline
\end{tabular}

Statistically significant values $(p<0.05)$ are in bold

* Student's $t$ test for paired samples 